\title{
Editorial
}

\section{Fault Detection, Isolation, and Tolerant Control of Vehicles using Soft Computing Methods}

Research on fault detection and isolation (FDI) and fault tolerant control (FTC), from both theoretical and practical aspects, has received more attention in recent years. The latest results confirm that there still remain some challenging areas within FTC/FDI on methodologies and computational complexities, as well as the implementation for a large domain of applications such as automobile, civil transportation airplanes, unmanned aerial vehicles, launch vehicles and satellites. Therefore, soft computing methods have attracted considerable attention from both the academic and industrial communities, emerging globally into various control applications. They have shown to be effective approaches for the FDI/FTC of many complex systems, including nonanalytic systems. Moreover, numerous advanced ideas in FDI/FTC methodology, including neural network and fuzzy approaches, have been proposed. Among various modelbased fuzzy-control strategies, the Takagi-Sugeno (T-S) method is extensively exploited for model-based nonlinear control designs. Indeed, significant efforts have been devoted to stability analysis, controller/observer design and FDI/FTC methods of these dynamic systems.

This Special Issue focuses on theoretical and practical aspects on new and emerging trends in FDI/FTC with vehicles applications using fuzzy logic and neural networks. It contains twelve papers, the contents of which are summarised below.

Jian et al. proposed a theoretical FTC framework for reconfigurable flight control in their paper entitled 'Adaptive neural observer-based backstepping fault tolerant control for near space vehicle under control effector damage'. Under this framework, two units are designed; one is an adaptivebased neural observer and the other is a reconfigurable controller which is based on an adaptive neural observer. A fault-tolerant control design based on the T-S fuzzy system models and terminal sliding mode control is investigated by $\mathrm{Xu}$ in his paper 'Study of T-S fuzzy-based terminalsliding-mode fault-tolerant control'. It is shown that this hybrid scheme can keep the advantages of both methods. Fan et al. studies the speed trajectory tracking problem of high-speed trains with actuator failures and unknown speed delays, as well as control input saturations, in their paper entitled 'Iterative learning and adaptive fault-tolerant control with application to high-speed trains under unknown speed delays and control input saturations'. They propose a new adaptive iterative learning fault tolerant control strategy without the need for precise system parameters or analytically estimating bound on actuator failures variables. Shen et al. investigated the finite-time fuzzy-model-based reliable control problem for a class of nonlinear systems in their paper 'Finite-time reliable $\mathcal{L}_{2}-\mathcal{L}_{\infty} / \mathcal{H}_{\infty}$ control for Takagi-Sugeno fuzzy systems with actuator faults'. The aim is to design a Markov switching fuzzy controller such that the resulting closed-loop system is stochastically finite-time bounded and satisfies a mixed disturbance attenuation over a finite time interval. Zhu and Xia studied the problem of fault detection for discrete systems with control inputs, unknown bounded disturbances and sensor faults in their paper entitled 'Fault detection for discrete systems based on descriptor system method'. The paper aims to design an observer-based fault detection filter such that the error dynamic system is convergent; the effect of the disturbances on the residuals satisfies the $\mathrm{H}_{\infty}$ performance index; and the effect of the faults on the residuals satisfies the $\mathrm{H}_{-}$performance index.

From an application point of view, Wang et al. proposed a passive FT control scheme for over-actuated systems to preserve the closed-loop stability in spite of different types of actuator faults in their paper 'Linear parametervarying based fault-tolerant controller design for a class of over-actuated nonlinear systems with applications to electric vehicles'. It is shown that the designed FT controller has no limitation on the control effect distribution ratios for the actual control effects, which allows the control system to distribute the higher-level control efforts to the lowerlevel actuators without using a control allocation algorithm. Zhao et al. proposed a novel approach for fault-tolerant flight control systems in their paper entitled 'Modeling and fault tolerant control for near space vehicles with vertical tail loss'. This paper mainly solved two key problems: it first establishes an analytical model for a damaged vertical tail; and secondly, in light of the damaged model, a RBF $\mathrm{NN}$ backstepping controller, which could greatly reduce the possibility of catastrophic accidents, is designed.

Aouaouda et al. investigated the issues of the descriptor approach and multiple Lyapunov functions leading to strict LMIs based on robust fault estimation and a fault tolerant controllers design for the class of continuous-time disturbed $\mathrm{T}-\mathrm{S}$ systems In their paper entitled 'Robust static output feedback controller design against sensor failure for vehicle 
GUEST EDITORS

HAMID REZA KARIMI

dynamics'. It is demonstrated, through a vehicle nonlinear model, how this modeling approach is effective to deal with unmeasured premise variables for FTC design.

$\mathrm{Li}$ et al. studied the problem of output-feedback $\mathrm{H}_{\infty}$ control for T-S fuzzy systems with input time-varying delay in their paper 'Fuzzy output-feedback control for nonlinear systems with input time-varying delay'. Firstly, a new type of dynamic output-feedback controller is constructed for fuzzy systems with input time-varying delay. Secondly, using the Lyapunov stability theory, a condition with $\mathrm{H}_{\infty}$ performance is developed. Sun et al. proposed a fault tolerant control approach to deal with the problem of fault accommodation for unknown actuator failures of active suspension systems in their paper entitled 'Reliability control for uncertain half-car active suspension systems with possible actuator faults'. Also, the generalised H2 fault detection problem for a class of discrete-time switched systems with repeated scalar nonlinearities is investigated by Zheng et al. in their paper 'Fault detection of switched systems with repeated scalar nonlinearities'. Finally, in 'Global consensus of single-integrator agents subject to saturation constraints', Wang et al. studied the problem of leaderfollowing consensus of single-integrator agents subject to saturation constraints.

While the selected topics and papers are not a comprehensive representation of the area of this Special Issue, they offer rich and multifaceted knowledge that we have the pleasure of sharing with readers.

Finally, we would like to thank all the authors for contributing submissions to the Special Issue and acknowledge all the reviewers for their voluntary work. We also thank the Editorial Office and Editor-in-Chief of IET Control Theory \& Applications, for their great support to this project, without which, the completion of this work would not be possible.
Faculty of Engineering and Science,

University of Agder, N-4898 Grimstad, Norway

hamid.r.karimi@uia.no

MOHAMMED CHADLI

University of Picardie Jules Verne, Laboratory of Modeling, Information \& Systems, Amiens, France mohammed.chadli@u-picardie.fr

PENG SHI

School of Electrical and Electronic Engineering, The University of Adelaide, SA 5005, Australia

College of Engineering and Science, Victoria University, Melbourne, Vic 8001, Australia Peng.Shi@vu.edu.au

LIXIAN ZHANG

Space Control and Inertial Technology Research Center, Harbin Institute of Technology, Harbin, P.R. China lixianzhang@hit.edu.cn 